\title{
EARLY EXTERNAL CEPHALIC VERSION 2
}

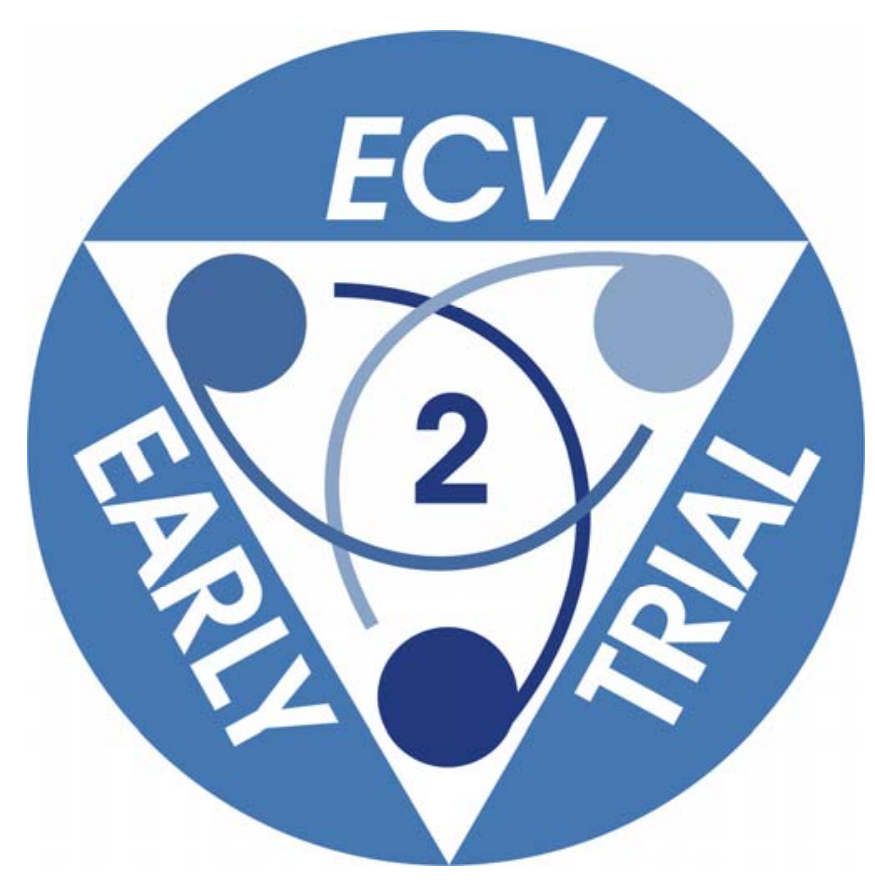

TRIAL PROTOCOL

February 2007 


\section{EARLY EXTERNAL CEPHALIC VERSION 2}

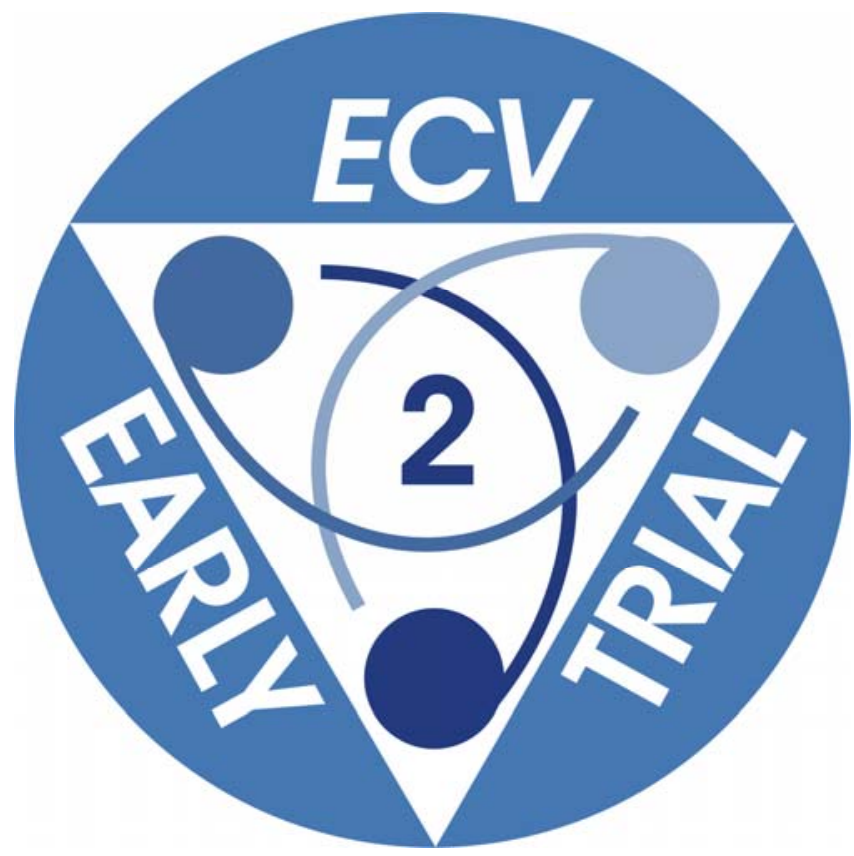

TRIAL PROTOCOL

February 2007

Data Coordinating Centre

University of Toronto

Maternal, Infant and Reproductive Health Research Unit

790 Bay Street, $7^{\text {th }}$ Floor, Toronto ON

Canada M5G 1N8

TEL: 416-351-3787

FAX: 416-351-3771

Email: eecv2@sw.ca 


\section{EARLY EXTERNAL CEPHALIC VERSION (ECV) 2 TRIAL}

\section{Table of Contents}

\begin{tabular}{|c|c|}
\hline 1 & THE NEED FOR A TRIAL \\
\hline 1.1 & What is the problem to be addressed? \\
\hline 1.2 & What are the principal research questions to be addressed in the Early ECV 2 Trial? \\
\hline 1.2 .1 & Primary Research Question \\
\hline 1.2 .2 & Secondary Research Question \\
\hline 1.2 .3 & Other Research Questions \\
\hline 1.3 & Why is a trial needed now? \\
\hline 1.4 & Systematic reviews and need for the trial in light of these reviews. \\
\hline 1.5 & How will the results of this trial be used? \\
\hline 1.6 & Risks to the safety of participants involved in the trial \\
\hline 2 & THE EARLY ECV 2 TRIAL PROTOCOL ......... \\
\hline 2.1 & What is the proposed research design? \\
\hline 2.2 & What is the planned trial intervention? \\
\hline 2.2 .1 & Timing of randomisation \\
\hline 2.2 .2 & Prior to randomisation \\
\hline 2.2 .3 & Early ECV \\
\hline 2.2 .4 & Delayed ECV \\
\hline 2.2 .5 & Both groups \\
\hline 2.3 & What are the proposed practical arrangements for allocating women to trial groups? \\
\hline 2.4 & What are the proposed methods of protecting against sources of bias? \\
\hline 2.4.1 & Contamination \\
\hline 2.4 .2 & Co-intervention \\
\hline 2.5 & What are the planned selection criteria? \\
\hline 2.5 .1 & Inclusion criteria for participants \\
\hline 2.5 .2 & Exclusion criteria for participants \\
\hline 2.5 .3 & Selection criteria for participating centres \\
\hline 2.6 & What is the proposed duration of the treatment period? \\
\hline 2.7 & What is the proposed frequency and duration of follow-up? \\
\hline 2.8 & What are the proposed outcome measures? \\
\hline 2.8 .1 & Primary outcome: CS \\
\hline 2.8 .2 & Secondary outcome: preterm birth (<37 weeks gestation) \\
\hline 2.8 .3 & Other outcomes \\
\hline 2.9 & How will outcome measures be measured at follow-up? \\
\hline 2.10 & Will health services research issues be addressed? \\
\hline 2.10 .1 & Economic evaluation \\
\hline 2.10 .2 & Other health services research issues \\
\hline 2.11 & What is the proposed sample size? \\
\hline 2.12 & What is the planned recruitment rate? \\
\hline 2.13 & Are there likely to be any problems with compliance? \\
\hline 2.14 & What is the likely rate of loss to follow up? \\
\hline 2.15 & How many centres will be involved? \\
\hline 2.16 & What is the proposed type of analysis and frequency? \\
\hline 2.16 .1 & Interim analysis \\
\hline 2.16 .2 & Final analysis \\
\hline 2.16 .3 & Subgroup analysis \\
\hline 2.17 & Has any pilot study been carried out using this design? \\
\hline 2.18 & Timetable for the study \\
\hline 3 & TRIAL MANAGEMENT . \\
\hline 3.1 & Arrangements for day-to-day management of the trial \\
\hline 3.2 & The role of each applicant \\
\hline 3.3 & The Steering Committee and the DSMB \\
\hline & DF \\
\hline
\end{tabular}




\section{EARLY EXTERNAL CEPHALIC VERSION (ECV) 2 TRIAL PROTOCOL - Revised February 2007}

\section{THE NEED FOR A TRIAL}

\subsection{What is the problem to be addressed?}

About 3-4\% of all pregnant women who reach full term will have a fetus presenting by the breech. The majority of these women would prefer a vaginal birth (VB) ${ }^{1-3}$ although most would choose Caesarean section (CS) if there is a medical indication. ${ }^{4}$ For the singleton fetus in breech presentation, $\mathrm{CS}$ has been shown to be safer for the fetus than $\mathrm{VB},{ }^{5-8}$ and the majority of care providers now recommend CS for breech pregnancies. ${ }^{9}$ The risks associated with CS are low, but CS is not without maternal risk and remains the largest contributing factor to the incidence of maternal mortality and morbidity following childbirth. ${ }^{10} \mathrm{CS}$ (elective and emergency) is estimated to quadruple the risk of severe morbidity compared to VB. ${ }^{11-13}$ In Canada in 1995-98 the three month readmission rate to hospital following birth (a proxy for complications of childbirth) was 3.9\% for CS, compared to $2.6 \%$ for VB. ${ }^{14}$ An American study also found increased rates of rehospitalisation following CS, with a relative risk of 1.8 compared to $\mathrm{VB} .{ }^{15}$ It has been argued that outcomes may be different for elective versus emergency CS. ${ }^{10}$ However, a Cochrane review of planned CS versus planned vaginal delivery for breech pregnancy at term, reported that even though $45 \%$ of women in the planned vaginal delivery group were delivered by CS, planned CS was associated with an increase in maternal morbidity (RR $[95 \% \mathrm{CI}]=1.29[1.03,1.61]){ }^{8}$ Furthermore, recent estimates of the incidence of mortality associated with elective CS were nearly tripled compared to VB. ${ }^{11,12}$ In addition to the increase in immediate morbidity following CS, intra-abdominal adhesions may occur after CS resulting in subsequent infertility. ${ }^{16}$ Also, the presence of the uterine scar puts future pregnancies at increased risk of complications such as ectopic pregnancy, placenta previa, accreta and abruption, and uterine rupture. $^{10,17-19}$ In Canada the CS rate in 2001 was $19.2 \%$, and in $199710 \%$ of all pregnant women had a prior CS scar. ${ }^{20,21}$ A further deterrent to CS is that the procedure requires the expertise of an obstetrician or other physician with surgical training, and limits the role for low risk obstetrical care providers such as midwives and family practitioners.

A review of strategies to reduce $C S$ rates identified external cephalic version $(E C V)$ as the only clinical intervention with demonstrated Level 1 evidence for reducing primary CS rates overall. ${ }^{22} \mathrm{~A}$ Cochrane review reported that ECV at term ( $\geq 37$ weeks) will increase the likelihood that the fetus will be cephalic at delivery, and will lower the need for $\mathrm{CS}^{23} \mathrm{ECV}$ is recommended for all women with a breech fetus at term, where there is no contraindication. ${ }^{24}$ However the procedure is often unsuccessful, particularly in North American and European settings. ${ }^{23,25}$ Although $89 \%$ of Canadian practitioners reported that they recommend ECV for breech pregnancies, their estimated success rate for ECV was $30 \%$ for nulliparous and $58 \%$ for multiparous women. ${ }^{9}$ A Cochrane Review of ECV begun before term found that there may be benefit to beginning ECV early (between 34-35 weeks) compared to beginning at term and recommended that further trials are needed. ${ }^{26}$

The Early ECV Pilot Trial (CIHR grant MT-15223) was undertaken to determine if beginning ECV somewhat earlier than term (34-35 weeks) might be more effective than beginning ECV at term (37-38 weeks). ${ }^{27}$ The objective of the Early ECV Pilot Trial was to determine if the rate of non-cephalic presentation at delivery could be reduced by beginning the procedure earlier, with the view that if this occurred, a larger trial would then be needed to evaluate the earlier procedure in terms of its impact on rate of CS and on adverse fetal and neonatal outcomes. The rate of non-cephalic presentation at birth in the early ECV group was 66/116 (56.9\%) and 77/116 (66.4\%) in the delayed ECV group (RR [95\% CI] $=0.86[0.70,1.05], \mathrm{p}=0.09)$. The rate of CS in the early ECV group was 75/116 (64.7\%) and 83/116

\footnotetext{
${ }^{\mathrm{i}} \mathrm{ECV}$ is a procedure undertaken to manually turn the fetus from breech to cephalic presentation by manipulating the fetus through the maternal abdomen 
$(71.6 \%)$ in the delayed ECV group $(\mathrm{RR}[95 \% \mathrm{CI}]=0.90[0.76,1.08] \mathrm{p}=0.32)$. Neonatal outcomes were not different in the two groups. The rate of reversion to non-cephalic was low in both groups. The majority of women in both groups indicated that they would consider having an ECV in another pregnancy. There was a clinically important reduction in the rate of both non-cephalic presentation at delivery (9.5\%) and of CS (7\%) in the early versus the delayed ECV group. ${ }^{27}$ These promising results indicate that beginning ECV at 34-35 weeks may be more effective than beginning ECV at term. However, the findings need to be confirmed in a larger trial and fetal safety with the earlier procedure needs to be established, before a change in clinical practice can be recommended. The Early ECV 2 Trial is thus proposed.

\subsection{What are the principal research questions to be addressed in the Early ECV 2 Trial?}

1.2.1 Primary Research Question For women with a fetus in breech presentation, does early ECV (at $34^{0 / 7}-35^{6 / 7}$ weeks) versus delayed ECV (not before $37^{0 / 7}$ weeks) increase or decrease the likelihood of CS?

1.2.2 Secondary Research Question Is the risk of preterm birth $\left(<37^{0 / 7}\right.$ weeks $)$ lower or higher with early versus delayed ECV?

1.2.3 Other Research Questions What is the effect of early versus delayed ECV on: a) admission to the neonatal intensive care unit for $\geq 24$ hours? b) perinatal or neonatal mortality or serious neonatal morbidity? c) serious fetal complications? d) maternal death or serious maternal morbidity? e) non-cephalic presentation at birth? f) pain experienced during the ECV procedure? g) women's views regarding the timing and use of ECV as an approach for their care? and h) health care costs?

\subsection{Why is a trial needed now?}

The prevalence of breech presentation in otherwise uncomplicated term pregnancies is 3-4\%, and as a result of the implementation of the Term Breech Trial findings, nearly all will result in CS. 5,9 Most women wish to avoid CS, and ECV is the only effective intervention to convert a breech fetus to cephalic presentation with the potential to help women avoid CS. ${ }^{1-3,23}$ Studies of ECV at term from Africa report very high rates of success of ECV ranging from $82-97 \%,{ }^{28,29}$ but these findings have not been replicated in studies from North America and Europe where success rates have ranged from 36$68 \%$. It has been suggested that differences in pelvic structure may allow the fetus to engage into the pelvis earlier in women of European descent making ECV more difficult to accomplish. ${ }^{30-33}$ The Early ECV Pilot Trial compared ECV that was started earlier (at 34-35 weeks gestation) to the usual time (3738 weeks) for nulliparous women with any breech presentation and for multiparous women with a frank breech presentation. This pilot study found that beginning ECV early decreased the non-cephalic presentation rate by $9.5 \% .{ }^{27}$ This result is now likely to translate directly into a reduction in the rate of CS due to the implementation of the Term Breech Trial findings. ${ }^{9}$ If the rate of CS could be decreased by $10 \%$ by using early ECV, the number needed to treat (NNT) to prevent $1 \mathrm{CS}$ would be 10 . This means that only 10 women would need to have an ECV performed early instead of having an ECV at $\underline{\text { term }}$ in order to avoid $1 \mathrm{CS}$. The results of the Early ECV Pilot Trial are therefore promising and suggest that there may be a real benefit to beginning the procedure early. However the results may have been due to chance (RR of non-cephalic presentation at birth $[95 \% \mathrm{CI}]=0.86[0.70,1.05] \mathrm{p}=0.09)$ and although there were no differences found in the pilot trial, increased risk of preterm birth and fetal

problems cannot be ruled out. ${ }^{27}$ Practitioners have indicated an interest in beginning ECV early. ${ }^{34-37}$ It will be important to undertake a large trial now, before early ECV is implemented into practice, to ensure that the benefits outweigh risks and establish whether early ECV will result in decreased CS without risk to the fetus or neonate. 


\subsection{Systematic reviews and need for the trial in light of these reviews.}

Four relevant Cochrane systematic reviews have been identified which support the development and conduct of the trial.

Planned CS for term breech delivery ${ }^{8}$ This review included three trials that compared planned CS to planned VB for breech presentation. ${ }^{5}$ The results indicate that planned CS greatly reduced both perinatal/neonatal mortality and neonatal morbidity, at the expense of a modest increase in maternal morbidity. The review thus supports the view that CS is the optimum method of delivery for breech fetuses, and strengthens the argument that a management practice that would turn a fetus from breech to cephalic, is likely to be the best way to avoid CS.

ECV for breech presentation at term ${ }^{23}$ This review included six RCTs of ECV at term versus no ECV in women with breech presentation. It concluded that ECV at term was associated with a significant reduction in non-cephalic births (RR $0.42,95 \%$ CI 0.35 to 0.50 ) and CS (RR $0.52,95 \%$ CI 0.39 to 0.71 ). It found no significant effect on perinatal mortality, but noted that there was insufficient evidence to assess the risks of ECV at term. Thus the review confirmed the value of conducting ECV at term. It is for this reason that ECV at $\geq 37$ weeks is the usual standard of care against which early ECV will be compared in the proposed Early ECV 2 Trial.

Interventions to help ECV for breech presentation at term ${ }^{38}$ This series of reviews examined the use of routine tocolysis, fetal acoustic stimulation, epidural or spinal analgesia, and transabdominal amnioinfusion for ECV at term, and their effect on successful version and pregnancy outcome. For each of these reviews, the comparison was against no or dummy treatment. In six trials, routine tocolysis was associated with fewer failures of ECV (RR $0.74,95 \%$ CI 0.64 to 0.87 ) and reduction in CS (RR 0.85 , $95 \%$ CI 0.72 to 0.99 ), but no difference in presentation at birth. The tocolytic review concluded that there was benefit from using tocolysis, but did not take into account the uncomfortable side effects (for example, palpitations and headache) experienced by many women. ${ }^{39}$ The reviews identified only three trials of regional anaesthesia, one trial of fetal acoustic stimulation, and no trials of transabdominal amnioinfusion. The reviews of these studies concluded that there was insufficient evidence to support the use of any of these treatments. These reviews have informed the development of our protocol for provision of ECV, in which we recommend that tocolysis should be considered for all women in the trial (Section 2.2.5), but do not recommend the other interventions in the protocol for the proposed Early ECV 2 Trial.

ECV for breech presentation before term ${ }^{26}$ This review included 3 trials. ${ }^{27,40,41}$ Two of the studies compared ECV to no ECV beginning prior to term. The third, which was the pilot study for the EECV2 Trial, compared ECV beginning at 34-35 weeks with ECV beginning after 37 weeks gestation. The review concluded that there may be some benefit to beginning ECV between 34-35 weeks gestation in terms of decreasing the rate of non-cephalic presentation, and caesarean section. Further trials were recommended to confirm benefit and rule out increased rates of preterm birth or other adverse perinatal outcomes.

\subsection{How will the results of this trial be used?}

The majority of women prefer to have a VB..$^{1-3}$ Women who decide to have a CS, do so on the basis of previous obstetrical complication, or increased obstetrical risk in the current pregnancy. ${ }^{4}$ Based on the best current evidence, women who present with a breech fetus will be advised to have a CS. ${ }^{5-9}$ ECV is considered the best treatment option to avoid a CS in these circumstances. ${ }^{23}$ However, the median self-estimated success rate of ECV at term amongst Canadian obstetric care providers for nulliparas was only $30 \%$ and for multiparas was $58 \% .{ }^{9}$ An approach that would reduce the rate of CS without injury to the fetus would be useful in clinical practice. The Early ECV 2 Trial will determine if early ECV is a better option than ECV at term in order to avoid CS, and will therefore allow clinicians, women, and policy makers to make a more informed choice about the ideal management of breech presentation. 
Many practitioners and women wish to reduce the rate of CS and are interested in ECV as a possible approach. ${ }^{36,37}$ The results of the Early ECV 2 Trial will be timely in determining the best obstetrical approaches to the management of breech pregnancies.

\subsection{Risks to the safety of participants involved in the trial}

In this study, women will undergo careful evaluation of the pregnancy with ultrasound prior to considering ECV, and will be randomised to one of two procedures that are used in current practice. ECV at $\geq 37$ weeks is the usual standard of care in the centres involved in the trial, and therefore women and babies in the delayed ECV group will not be exposed to additional risk over and above usual care. The results from the Early ECV Pilot Trial did not suggest an increased risk for mothers with early ECV compared to delayed ECV. Pain experienced by the mothers did not differ significantly between groups. The rate of preterm birth $<37$ weeks, and the rate of serious fetal complications were not significantly increased in the early ECV group compared to the delayed ECV group $(8.6 \%$ vs. $6.1 \%, p=0.31$, and $6.9 \%$ vs. $7.8 \%, p=0.69$ respectively). However, because the trial was not designed to examine these outcomes, the confidence intervals were wide and important decreases or increases in risk may have been missed. Adverse neonatal outcomes were infrequent in both groups. Although the findings from the Early ECV Pilot Trial were reassuring, there is less experience with early ECV and therefore more concern. For this reason we have included an interim analysis to examine safety (see Section 2.16.1).

Regardless of when the ECV procedure is undertaken there is likely an increased risk of fetomaternal haemorrhage. Lau et al found the risk of a fetomaternal haemorrhage $>4 \mathrm{ml}$ after ECV at term to be $1.8 \%$ and recommended that $500 \mathrm{IU}$ of anti-D immunoglobulin be given to non-sensitised $\mathrm{Rh}$ negative women, followed by additional anti-D immunoglobulin if a Kleihauer test indicated the fetomaternal haemorrhage to be larger than $4 \mathrm{ml}^{46}$ We have included this recommendation in the protocol for the Early ECV 2 Trial (Section 2.2.5).

\section{THE EARLY ECV 2 TRIAL PROTOCOL}

\subsection{What is the proposed research design?}

To eliminate selection bias, a RCT with prognostic stratification for centre and parity will be used. Randomisation will be centrally controlled using a telephone randomisation service at the University of Toronto Maternal, Infant and Reproductive Health Research Unit (MIRU) at the Centre for Research in Women's Health. Eligible and consenting women will be randomised by centre within parity groups $(0$ and $\geq 1)$ to the early ECV group or to the delayed ECV group.

\subsection{What is the planned trial intervention?}

2.2.1 Timing of randomisation To minimise the number of women that will have a breech fetus that changes to a cephalic presentation spontaneously prior to entry into the study, randomisation will be delayed until as close to the timing of a possible early ECV as possible. In order to allow time for booking of procedures, women may be randomised between $33^{0 / 7}-35^{6 / 7}$ weeks, but the ECV procedures will be initiated between $34^{0 / 7}-35^{6 / 7}$ weeks in the early ECV group and $\geq 37^{0 / 7}$ weeks in the delayed ECV group.

2.2.2 Prior to randomisation A screening ultrasound will be undertaken at $32^{0 / 7}-35^{6 / 7}$ weeks gestation and within one week of randomisation. The ultrasound will confirm the breech presentation and rule out contraindications to the procedure and to vaginal delivery (identification of any major life-threatening fetal anomalies, hyper-extended fetal head, severe oligohydramnios, severe polyhydramnios, or placenta previa). Eligible women (defined in 2.5.1 \& 2.5.2) will give informed consent prior to randomisation. After collecting baseline information, consenting and eligible women will be randomised to either early or delayed ECV groups.

2.2.3 Early ECV ECV will be undertaken in the early ECV group at $34^{0 / 7}-35^{6 / 7}$ weeks gestation and within 7 days following randomisation. 
2.2.4 Delayed ECV ECV will be undertaken in the delayed ECV group at or after $37^{0 / 7}$

weeks.

\section{Early ECV-2 Trial Schema}

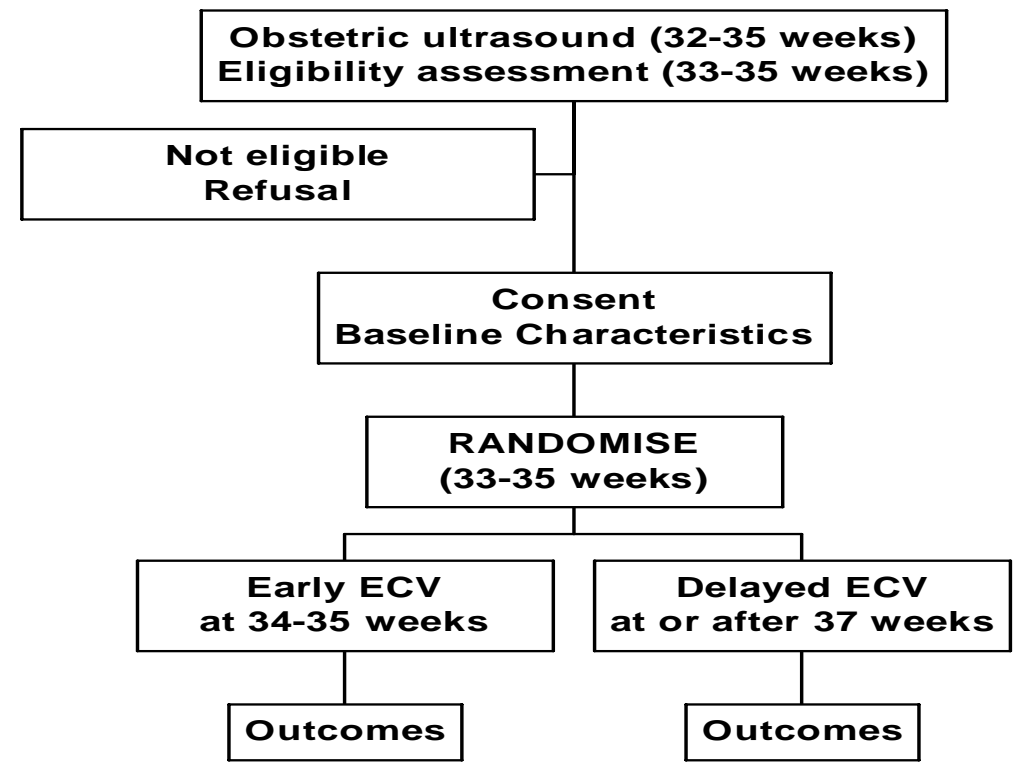

2.2.5 Both groups All ECV procedures will be undertaken by experienced clinicians (defined in 2.5.3) and in an environment where any complication can be appropriately managed.

Prior to beginning the procedure Immediately prior to the ECV procedure, the woman will be reassessed to ensure she is still eligible for ECV and the fetal presentation will be confirmed by ultrasound. This ultrasound is used along with clinical assessments to determine any contraindications to ECV prior to each procedure. Fetal well-being should be assessed prior to the procedure by continuous fetal heart rate monitoring for 20 minutes. Fetal heart rate assessment should reveal a normal baseline rate, good variability, and no evidence of decelerations prior to ECV.

Description of the procedure The procedure usually begins with the woman in a comfortable position lying on her back with legs slightly flexed at the knee. Arms should be extended and lying along side the woman in order to enhance abdominal relaxation. The clinician begins by palpating the fetus to ascertain the position of the cephalic pole and the fetal back. ${ }^{\text {ii }}$ The next step involves lifting the breech out of the pelvis and to one side, usually on the side opposite the cephalic pole. ${ }^{\text {iii }}$ When the fetus has been manoeuvered out of the pelvis, it is often useful to have a second attendant support the breech pole in that position, thereby minimizing the likelihood of the breech returning to the pelvic area. The fetus will usually turn most easily in a forward somersault. Once the breech has been successfully dislodged from the pelvic basin, the clinician will encourage the fetal head downward toward the pelvis. The fetus should respond to firm but gentle pressure by moving through the uterine midpoint and into a cephalic presentation. Follow up palpation should be done to confirm that the fetus has moved into a cephalic presentation, and not merely returned to its previous presentation. Fetal well-being will be monitored intermittently during the ECV attempt using auscultation, Doppler, or ultrasound viewing of fetal heart rate.

Duration of the procedure When undertaking the ECV procedure, the practitioner may pause for varying periods of time, to assess the fetal heart sounds, to allow the mother to relax her abdominal

\footnotetext{
ii If the fetus is in a posterior position it may be helpful to place the woman on hands and knees and ask her to pelvic tilt for 3-5 minutes. This often facilitates bringing the fetus into a more anterior position which favours the success of ECV.

${ }^{\text {iii }}$ Ranney describes a step in the ECV procedure of lifting the deeply engaged fetus out of the pelvis by way of the vagina. EECV2 Protocol Revised February 2007 
muscles, or to allow the fetus to settle into the new position. Because of the breaks in the procedure, the total time for the procedure may vary.

Unsuccessful attempts If a forward somersault does not prove successful, a manipulation in the backward direction may be attempted. With a backward somersault manoeuvre, the primary pressure should be asserted against the fetal breech, in order to enhance flexion of the fetal spine.

Tocolytics Evidence from a Cochrane review of tocolytics for ECV at or near term found a significant reduction in the failure rate of ECV with the use of tocolytics, and it is recommended that they be considered as potentially useful to relax the uterus for all women having ECV. ${ }^{38}$ Tocolytics may be used either for all women, or just for those where the practitioner anticipates that the ECV may be more difficult. Tocolysis is not mandated in the protocol, because many women experience uncomfortable side effects (palpitations, headache, etc), and some clinicians prefer to use tocolytics only when clearly indicated. ${ }^{39}$ If the preferred approach is to give tocolytics to all women having ECV, this should be done regardless of the timing of the procedure. Similarly, if the preferred approach is to use tocolytics only for selected women having ECV, this should be done regardless of the timing of the procedure. The health care provider will determine the choice of tocolytic, if used, and its method of administration.

Regional analgesia A Cochrane review of regional analgesia to facilitate ECV at term provided only limited evidence to support regional analgesia for $\mathrm{ECV},{ }^{38}$ therefore the decision to use regional analgesia in the trial will be left to practitioners. Since we are trying, in this study, to compare ECV at early and late gestational ages, and not ECV with or without regional analgesia, if a clinician chooses to use regional analgesia, this approach should be used in the same way for women in both arms of the trial.

For example, if regional analgesia is selectively used for the ECV procedure, when it is anticipated that the breech will be more difficult to turn, irrespective of gestational age, then it should be used selectively this way for both the early and delayed groups.

For example, if regional analgesia is used for the ECV procedure, routinely, when it is done at term, then it should be used routinely this way whenever an ECV procedure is done at term in either the early or delayed groups.

Discontinuing the procedure The procedure should be discontinued if fetal heart tones are nonreassuring, if it is not easily accomplished, or if the woman reports undue discomfort.

Following the procedure The woman will remain under supervision for at least one half hour following the procedure. Fetal well-being will be assessed following the procedure by confirming fetal movement on ultrasound and by recording a reactive fetal heart rate on continuous fetal heart rate monitoring. Fetal presentation should be confirmed by ultrasound immediately following the procedure.

Repeat procedures The ECV procedure may be repeated if necessary at the discretion of the care provider and in consultation with the woman.

Mode of delivery Based on the evidence from the Term Breech Trial that CS is the preferred mode of delivery for the singleton fetus in breech presentation at term, CS will be recommended to all women with a fetus that remains non-cephalic at the time of birth, and these pregnancies should be delivered by emergency CS if labour begins spontaneously or if there is an indication for urgent delivery. In the instance of rapidly progressing labour, every effort should be made to proceed to CS. All fetuses that are cephalic at birth and where there is no indication for CS, will be delivered vaginally. All other aspects of care during pregnancy, labour, and delivery will be determined by the woman and her caregiver.

Rhesus negative women Because Rhesus isoimmunization is a risk of $\mathrm{ECV}$, non-sensitised $\mathrm{Rh}$ negative women will be provided with anti-D immunoglobulin following the procedure. ${ }^{46}$

\subsection{What are the proposed practical arrangements for allocating women to trial groups?}


As nulliparity is associated with failure of ECV, randomisation will be stratified by parity to ensure that an equal number of nulliparous women are in each group. In order to control for possible differences in outcome between centres, randomisation will also be stratified by centre. Random block sizes will be used, to ensure that centres cannot anticipate the next allocation.

Randomisation will be centrally controlled using the computerised randomisation service at MIRU, which centres can access 24 hours per day using a toll-free telephone line. A back-up pager system will be maintained at all times to ensure availability of trial staff to handle situations where a technical difficulty is encountered, or where questions arise regarding trial entry. In situations where the automated system fails, hand randomisation will be undertaken.

\subsection{What are the proposed methods of protecting against sources of bias?}

A centrally controlled computerised randomisation service will be used (as described in 2.3). It is not possible to blind physicians or patients to the intervention. The primary outcome is objective, and all other outcomes have been defined to be as objective as possible. To avoid bias in assessing gestational age at delivery, gestational age will be determined prior to randomisation and this gestational age will be used in determining gestational age at delivery and the rate of preterm birth. Members of the Steering Committee will review stillbirths and neonatal deaths associated with fetal anomaly, blinded to allocation group, to determine if the anomaly is incompatible with life. Lethal anomalies will be excluded from the analysis of perinatal/neonatal outcomes.

2.4.1 Contamination Contamination of the delayed ECV group with early ECV is not likely to be a problem, as early ECV is not part of the usual obstetrical standard of care. The data will be monitored, however, to ensure this does not occur. If the fetus persists as breech until term in the early ECV group, ECV may be repeated at that time. The information provided to all potential participants and care providers involved in the trial will emphasize the importance of following the trial protocol after entry in the study.

2.4.2 Co-intervention The most important co-intervention is the use of tocolytics for ECV as they are known to increase the success of the procedure. ${ }^{38}$ Tocolytics have been recommended as part of the trial protocol. Centres are urged to use a similar approach to their use in both the early and delayed ECV groups. There is less evidence to support regional analgesia for ECV, and decisions around use will be left to practitioners. Ultimately the results will represent the ECV procedure as used in this study; that is with or without tocolytics and regional analgesia. A further potential for cointervention is the use of common alternative modalities for promoting spontaneous version of the fetus, such as pelvic tilting exercises, knee chest position, chiropractic adjustments, acupuncture and increasing fluid hydration. This is unlikely to have an impact on the study results, as there is no substantial evidence that these approaches are effective at altering fetal presentation. ${ }^{38,47}$

\subsection{What are the planned selection criteria?}

The target population consists of pregnant women with a singleton fetus in a breech presentation who are at 34-35 weeks gestation. ${ }^{\text {iv }}$ An ultrasound should be performed within 7 days before randomisation to confirm a live fetus in breech presentation, and to rule out contraindications to ECV, to early ECV, or to labour or vaginal delivery. Because of challenges involved in booking procedures, women may be enrolled as early as 33 weeks gestation, but no ECV will be undertaken before 34 weeks gestation.

\subsubsection{Inclusion criteria for participants}

1. women with any breech presentation

2. live singleton fetus

3. gestational age $33-35$ weeks

iv 34 weeks $=34$ weeks 0 days to 34 weeks 6 days 


\subsubsection{Exclusion criteria for participants}

1. contraindications to ECV (e.g. fetal heart rate abnormalities, abruptio placenta, major life-threatening fetal anomalies, uterine anomalies, hyper-extended fetal head, rupture of membranes, severe

oligohydramnios, severe polyhydramnios)

2. contraindications to early ECV (e.g. increased risk of preterm labour [such as past history of preterm labour in this or previous pregnancies], increased risk of abruptio placenta [such as maternal hypertension])

3. contraindications to labour or vaginal delivery (e.g. placenta previa, previous classical CS)

4. women planning delivery by CS if the fetus turns to cephalic

5. women planning a vaginal delivery if the fetus remains breech

6. women at increased risk of unstable lie (eg. grand multiparity)

7. previous participation in EECV2 Trial

2.5.3 Selection criteria for participating centres In order for the trial to have clinical relevance it is important to establish that the ECV is undertaken or supervised by clinicians who have adequate experience with the procedure. We will use the following definition of experience which was adapted from the Term Breech Trial, and which has been demonstrated to be robust: clinicians will be eligible to participate if they judge themselves to be skilled and experienced in the ECV procedure and their Head of Department agrees with this judgment. ${ }^{51}$ Participating centres must be able to ensure that an experienced clinician will undertake all ECV procedures, and that they have the anaesthetic, obstetrical and nursing staff to be able to undertake a CS, if necessary, usually within 30 minutes of making the decision to do so.

Participating centres must be able to provide the following care for infants enrolled in the Early ECV 2 Trial: suitable facilities and qualified neonatal staff who are able to resuscitate a depressed baby by giving oxygen [by mask, bag and mask or ventilator], provide ventilation by endotracheal intubation and positive pressure ventilation, give intravenous therapy and blood transfusion and use surfactant. Centres must be able to obtain a neonatal head ultrasound, if necessary.

\subsection{What is the proposed duration of the treatment period?}

The study treatment is ECV: the period of treatment is therefore the time taken to undertake ECV. ECV may be repeated as described (Section 2.2.5).

\subsection{What is the proposed frequency and duration of follow-up?}

Women and infants will be followed until 28 days after delivery or until hospital discharge, whichever is later.

\subsection{What are the proposed outcome measures?}

2.8.1 Primary outcome: CS The results of the Early ECV Pilot Trial demonstrated a promising trend towards lower rates of non-cephalic presentation at birth and CS. The Early ECV 2 Trial will be adequately sized to assess the effect of early versus delayed ECV in terms of the need for CS.

2.8.2 Secondary outcome: preterm birth ( $<37$ weeks gestation) Preterm birth impacts on neonatal morbidity ${ }^{49}$ and health care utilisation, and is therefore an important surrogate outcome for serious perinatal or neonatal morbidity.

\subsubsection{Other outcomes}

a) admission to the neonatal intensive care unit $\geq 24$ hours.

b) perinatal or neonatal mortality or serious neonatal morbidity. A composite measure of perinatal or neonatal mortality or serious neonatal morbidity ${ }^{\mathrm{v}}$ will be defined as one or more of:

\footnotetext{
${ }^{\mathrm{v}}$ It is recognized that some of these morbidities are not usually associated with a gestational age $>33$ weeks gestation $\begin{array}{lll}\text { EECV2 Protocol Revised February } 2007 & -8- & \text { Approved May } 2007\end{array}$
} 
1. Death (stillbirth or neonatal death 0 to 27 days after birth) (excluding lethal anomalies) Exclusions for reasons of lethal congenital anomalies will be determined without knowledge of group allocation.

2. Birth trauma defined by any of the following:

a) Spinal cord injury

b) Basal skull fracture or depressed skull fracture

c) Long bone fracture (humerus, radius, ulna, femur, tibia or fibula)

d) Peripheral nerve injury (brachial plexus palsies, phrenic nerve palsy, facial nerve palsy)

present at 72 hours of age (or at discharge from hospital if sooner)

e) Subdural or intracerebral haemorrhage of any kind (confirmed by ultrasound, computerised tomography [CT], magnetic resonance imaging [MRI], or at autopsy)

3. Apgar score at 5 minutes less than 4

4. Abnormal level of consciousness including:

a) Coma

b) Stupor or decreased response to pain

5. Neonatal seizures, defined as clonic movements which cannot be stopped by holding the limb, occurring on two or more occasions before 72 hours of age, regardless of cause

6. Need for assisted ventilation $\geq 24$ hours via endotracheal tube initiated within 72 hours after birth

7. Infection determined within $72 \mathrm{hrs}$ after birth, defined by either of the following:

a) Septicemia (positive blood culture)

b) Meningitis (positive cerebrospinal fluid culture)

8. Necrotising enterocolitis (defined as either perforation of intestine, pneumatosis intestinalis or air in the portal vein) diagnosed by X-ray, surgery, or at autopsy

9. Broncho-pulmonary dysplasia (BPD): Need for oxygen at a postnatal gestational age of 36 completed weeks as well as an X -ray compatible with BPD

10. Intraventricular hemorrhage (IVH): Grade III or IV (diagnosed by cranial ultrasound or at autopsy)

11. Cystic Periventricular Leukomalacia (PVL): Periventricular cystic changes in the white matter excluding subependymal and choroid plexus cysts (diagnosed by cranial ultrasound or at autopsy).

c) serious fetal complications. This is defined as one or more of the following:

i) Preterm prelabour rupture of the membranes

ii) Abruptio placenta requiring obstetrical intervention to effect delivery

iii) Preterm labour followed by preterm birth

iv) Abnormalities of the fetal heart rate prior to labour requiring obstetrical intervention to effect delivery

d) maternal death or serious maternal morbidity. This is defined as one or more of the following during pregnancy, labour, birth or within the first 28 days following the birth: death; haemorrhage (documented blood loss of $>1500 \mathrm{cc}$, blood transfusion required, or need for dilation/curettage or manual removal of the placenta after delivery); laparotomy excluding CS or tubal ligation; genital tract injury (hysterectomy, vulvar or perineal haematoma requiring evacuation, symptomatic broad ligament haematoma confirmed by ultrasound, CT or MRI, intraoperative damage to bladder, ureter or bowel requiring repair, fistula involving the genital tract, $3^{\text {rd }}$ or $4^{\text {th }}$ degree perineal tear involving the anal sphincter and/ or mucosa); thromboembolism (deep vein thrombosis, thrombophlebitis, or pulmonary embolism) requiring anticoagulant therapy; systemic infection (temperature of $38.5^{\circ} \mathrm{C}$ or more on 2 or more occasions at least 24 hours apart not including the first 24 hours, or pneumonia [confirmed by Xray], or sepsis [confirmed by blood culture]; major medical life threatening illness (e.g. adult respiratory distress syndrome, amniotic fluid embolism, disseminated intravascular coagulation, bowel obstruction, paralytic ileus [requiring nasogastric suctioning]); wound infection (requiring prolongation of hospital stay, readmission to hospital or repeated treatment as an outpatient), dehiscence, or breakdown; or other serious maternal complication.

e) non-cephalic presentation at birth 
f) pain experienced during the procedure for women having an ECV procedure: Women who have an ECV procedure will be asked to complete a single visual analog scale immediately following the procedure to rate the amount of pain experienced during the procedure.

g) women's views: Maternal satisfaction will be determined using a structured questionnaire that will ask women if, in a subsequent pregnancy, they would have the same approach for turning their breech baby as they had in this study, and whether they would recommend this approach to a friend if her baby was in breech presentation. Additional information will be sought from women regarding what they liked or disliked about ECV generally and specifically about the timing of the procedure. Women will also be asked about their feelings regarding taking part in a research trial.

h) health care costs: a cost analysis is planned for Canadian centres taking part in this trial, using a ministry of health perspective to determine if beginning ECV early will result in decreasing the CS rate and result in decreased health care costs. This evaluation is further described in Section 2.10.1.

\subsection{How will outcome measures be measured at follow-up?}

Information pertaining to $\mathrm{CS}$, preterm birth, presentation at birth, perinatal and neonatal outcomes, maternal outcomes, and serious fetal complications will be abstracted from women's medical records. Women will be contacted by telephone, or by home or clinic visit at 28 days following the birth, to confirm that they and their babies are well. All participants will be asked to complete a structured questionnaire in the first few days postpartum either prior to discharge or by phone, to determine satisfaction with the approach to their care.

\subsection{Will health services research issues be addressed?}

2.10.1 Economic evaluation The economic hypothesis for the study is that a policy of beginning ECV early will result in decreasing the CS rate, and will therefore result in decreased health care costs. Thus a cost analysis is planned for Canadian centres taking part in this trial, using a ministry of health perspective. Adding a cost component to the evaluation of the interventions will enable decision and policy makers to judge the relative merits of one intervention over the other. The cost evaluation will involve determining resources consumed and unit prices for these resources. The estimates of unit prices will be combined with the resource use information for all trial participants. Resources consumed in either intervention group in the Early ECV 2 Trial will be collected in the clinical case report forms. The estimation of unit prices in this trial will be obtained from a sample of Canadian participating centres. For the Early ECV 2 Trial, this sample will consist of six hospitals (three smaller/community hospitals and three larger/teaching hospitals).

The unit price estimation within each hospital will involve two stages. First, estimates of professional fees for outpatient doctor visits, ECV procedures, daily hospital visits, anaesthesia, and surgical operations will be obtained directly from each appropriate department/ministry of health (third party payer). Local health care experts will be asked to assist with the identification of the most appropriate fees for each service or course of treatment. Second, as existing per diem hospital rates are inadequate for detailed costing purposes, hospital cost models will be developed for each hospital. These models will be constructed with assistance and cooperation from hospital management and finance departments. The models will be designed to estimate department direct expenses, but will also include a share of hospital overhead expenses. Hospital unit price estimates, which exclude hospital overhead costs, may underestimate substantially the 'true' cost of hospital services. It is for this reason that considerable effort will be devoted to developing reliable and accurate hospital cost models. The estimates of unit prices will be combined with the resource use information for all trial participants.

2.10.2 Other health services research issues Women's satisfaction with the intervention and participation in the trial will be evaluated as described in $2.8 .3(\mathrm{~g})$.

\subsection{What is the proposed sample size?}


We estimate that the rate of CS in the delayed ECV group will be $65 \%$. This rate is somewhat lower than that found in the delayed ECV group of the Early ECV Pilot Trial (71.6\%) (where multiparas were only included if they had a frank breech fetus) because in the Early ECV 2 Trial we propose to include nulliparous and multiparous women with any breech presentation. This should result in a slightly higher rate of success with ECV in the delayed ECV group thus decreasing the CS rate in that group.

In order to determine what would be considered a clinically important decrease in CS rate with early ECV, we undertook a survey of site investigators to determine the minimum decrease in the rate of CS at which they would change their clinical practice. Survey results showed that $92 \%$ of practitioners would change practice based on a reduction in CS of $10 \%$, and $52 \%$ would change practice based on a reduction in CS of less than 10\% [range 1\%-8\%; median 5\%]. Based on these findings we wish the sample size to be adequate to detect an $8 \%$ reduction in CS rate, from $65 \%$ in the delayed ECV group to $57 \%$ in the early ECV group, if such a reduction exists. With a sample size of 610 /group (total $=1220$ ), we will have $80 \%$ power of finding an $8 \%$ reduction in rate of CS from $65 \%$ in the delayed group to $57 \%$ in the early group, $\alpha$ error of 0.05 (2- tailed). With a reduction of $8 \%$ in rate of CS, $12-13$ women would need to have an ECV early instead of having it at term in order to avoid $1 \mathrm{CS}$. That is, the number needed to treat (NNT) to prevent 1 CS would be 12.5.

The rate of preterm delivery among the delayed ECV group in the Early ECV Pilot Trial was $6 \%$. With a sample of 1220 in the proposed trial, we will have $>70 \%$ power to detect a clinically important increase from $6 \%$ to $10 \%$, and $>90 \%$ to detect an increase from $6 \%$ to $12 \%$ in the rate of preterm delivery ( $\alpha$ error of 0.05 [two-sided]) if such an increase exists. The trial will also be able to examine major changes in other clinical outcomes, for example we will have $>85 \%$ power to detect a 3 fold increase in the rate of perinatal/neonatal mortality and serious neonatal morbidity from $1.6 \%$ to $4.8 \%$ (based on the difference in a similar outcome found in the Term Breech Trial). The trial is not powered to detect small changes. Because we are proposing to recruit women at an earlier gestational age to facilitate the booking of procedures, we estimate that the rate of spontaneous cephalic version, development of a contraindication, or women declining the procedure prior to the ECV procedure in the early ECV group will be as high as $20 \%$. We have therefore increased the sample size by $20 \%$ to account for this. The final sample size is thus 730/group or a total of 1460 women.

Based on our experience with the Early ECV Pilot Trial, we estimate that non-compliance with the trial protocol and loss to follow-up will be low $(<1 \%)$. 
Table 1. Power calculations for varying effect sizes; sample size $=610 * /$ group; $\alpha$ error $(2-$ tailed $)=0.05$

\begin{tabular}{lccc}
\hline Outcome & $\begin{array}{c}\text { Rate in Delayed } \\
\text { ECV Group }\end{array}$ & $\begin{array}{c}\text { Rate in } \\
\text { Early ECV } \\
\text { Group }\end{array}$ & Power \\
\hline CS & $\mathbf{6 5 \%}$ & $\mathbf{6 0 \%}$ & $\mathbf{4 4 \%}$ \\
\hline Preterm Birth & $\mathbf{6}$ & $57 \%$ & $\mathbf{8 0 \%}$ \\
& & $55 \%$ & $\mathbf{9 5 \%}$ \\
\hline Perinatal/neonatal mortality or & $1.6 \%$ & $\mathbf{1 0 \%}$ & $\mathbf{7 3 \%}$ \\
\cline { 2 - 4 } serious neonatal morbidity & & $\mathbf{1 1 \%}$ & $\mathbf{8 8 \%}$ \\
\hline
\end{tabular}

This sample size has been calculated to include continuity correction

\subsection{What is the planned recruitment rate?}

After consultation with collaborators, modifications have been made to the Early ECV 2 Trial protocol to facilitate recruitment. These modifications include recruitment as early as 33 weeks gestation (versus 34 weeks in the Pilot); allowing up to 7 days between the initial ultrasound and randomisation (versus 4 days in the Pilot), and allowing up to 7 days between recruitment and the early ECV procedure if randomised to the early ECV group (versus 4 days in the Pilot). In addition, centres will be encouraged to establish a systematic approach to screening for breech pregnancies at 32-35 weeks gestation to ensure that breech presentations are detected during the gestational age window of eligibility. We are hopeful that the rates of recruitment may be higher in this trial than occurred in the Pilot, but because there is a tendency to overestimate recruitment rates ${ }^{50}$ we have estimated the recruitment rate for the Early ECV 2 Trial based on the actual recruitment rate in the Pilot of $0.25 \%$ of all births. Eighty centres have agreed to participate in the Early ECV 2 Trial with a cumulative birth rate of more than 300,000 annually. Based on the births per year at centres and the recruitment rate in the Pilot, we estimate that 750 women will be recruited per year. We have also assumed that recruitment will begin slowly in some centres, as not all centres will be ready to recruit as soon as the Data Co-ordinating Centre is able to open the Early ECV 2 Trial for recruitment generally, and that not all centres will ultimately prove to be successful at recruitment. We therefore estimate that we shall be able to recruit 1460 women over a period of 30 months (2.5 years).

\subsection{Are there likely to be any problems with compliance?}

The compliance with the protocol in the Early ECV Pilot Trial was excellent. However, we will check compliance with the timing of ECV procedures in the 2 randomised groups, quarterly, for the trial as a whole and by centre. If problems occur, we will work with the centres to resolve them.

\subsection{What is the likely rate of loss to follow up?}

Women enrolled in the trial may be followed from as early as 33 weeks gestation to 28 days following delivery. It is unlikely that women will move unexpectedly during this period of time, and women who know they will move to a non-trial centre prior to delivery, will not be invited to participate. Every effort will be made to obtain all outcome information on all women enrolled in the study. In the Early ECV Pilot Trial, only 1/233 (0.4\%) was lost to follow-up, and in the Term Breech Trial which recruited from 121 centres world-wide, only 5/2088 (0.2\%) were lost to follow-up for the principal outcomes. We anticipate minimal loss to follow up for the principal outcomes in this trial. 


\subsection{How many centres will be involved?}

The large sample required to adequately answer the research question will necessitate recruitment from at least 80 centres (see Section 2.11), and will require international collaboration in order to complete the trial in a timely fashion. Of the 80 centres who have expressed an interest in taking part most are from Canada or from countries with similar obstetrical care systems. If other centres are keen to take part, in addition to those who have already expressed an interest, we will encourage them to join the trial in order to be able to accrue the sample size in as short a time period as possible and minimise the trial costs.

\subsection{What is the proposed type of analysis and frequency?}

2.16.1 Interim analysis Two planned safety interim analyses will be conducted after complete data have been received on the first 500 women randomized, and again after complete data have been received on the first 900 women randomized, to ensure that the intervention is not associated with an increased risk of CS, preterm birth or perinatal or neonatal mortality or serious neonatal morbidity. The rates of CS, preterm birth, and perinatal or neonatal mortality or serious neonatal morbidity will be compared in the 2 randomised groups and reviewed by an independent Data Safety and Monitoring Board (DSMB) blinded to group assignment. If there is a significant increase in any of these outcomes at $p<0.005$ (1-tailed) in the early ECV group, the trial will be stopped. The trial will not be stopped early if a reduction in the rate of CS is found. This is because the full sample size will be required to be confident that important increases in preterm delivery have been ruled out.

2.16.2 Final analysis An intention to treat analysis will be conducted. Baseline data will be analysed descriptively for the women and their pregnancies in the 2 groups. The rate of the primary and secondary outcomes (CS and preterm birth $<37^{0 / 7}$ weeks) will be compared in the 2 groups using Fisher's exact test for $2 \times 2$ tables. If there are differences between groups in confounding variables (frank breech presentation, gestational age at randomisation, national perinatal mortality rate [PMR] of country) these will be controlled for in a logistic regression analysis. As the Early ECV 2 Trial will stratify for parity at randomisation, we anticipate that this variable will not differ between groups. A p value of $<0.05$ (2-sided) will indicate statistical significance for the primary and secondary outcomes. Rates of other outcomes (admission to the neonatal intensive care unit $\geq 24$ hours, perinatal or neonatal mortality or serious neonatal morbidity, serious fetal complications, maternal death or serious maternal morbidity, non-cephalic presentation at birth, and women's views) will also be compared between groups using Fisher's exact test for $2 \times 2$ tables. Pain experienced during the first ECV procedure will be compared between groups using a Student $t$ test. A p value of $<0.01$ (2-sided) will indicate statistical significance for these other outcomes. Relative risks and appropriate confidence intervals will also be used to report the effects of the intervention on each outcome.

2.16.3 Subgroup analysis For the purpose of hypothesis generation, logistic regression analyses will be used to test for interactions between baseline characteristics (parity [0 vs. $\geq 1$ ], type of breech [frank versus non-frank], gestational age at randomisation [33 - 34 weeks versus 35 weeks], and the national PMR of the country $[\leq 20 / 1000$ vs. $>20 / 1000]$ and treatment group for the primary and secondary outcomes (CS and preterm birth).

\subsection{Has any pilot study been carried out using this design?}

As described in Section 1.1 of the background, the Early ECV Pilot Trial was funded by CIHR to determine if there was merit to beginning ECV at 34-35 weeks gestation compared to beginning ECV at 37-38 weeks gestation. The primary outcome for the Early ECV Pilot Trial was the rate of non-cephalic presentation at birth, as it was felt to be important to determine that beginning ECV early had an effect on this outcome before mounting a trial large enough to look at the outcomes of CS and fetal/neonatal safety. The reductions in rate of non-cephalic presentation at birth and rate of CS are clinically 
important and the Early ECV 2 Trial is designed to further evaluate early ECV in terms of CS rate and rate of preterm birth.

The Early ECV Pilot has informed the design of the Early ECV 2 Trial in a number of ways:

- In order to increase the generalisability of the findings, we are including nulliparous and multiparous women with any breech presentation, and not only those with a frank breech presentation.

- The Early ECV Pilot Trial was started before the findings of the Term Breech Trial were available. The currently available evidence supports the use of CS for delivery of the fetus that is non-cephalic, we now recommend this method of delivery for breech fetuses in the Early ECV 2 Trial protocol, and women who are planning a vaginal breech birth will be excluded from the trial.

- Some centres that participated in the Early ECV Pilot Trial found it difficult to schedule ultrasound and ECV procedures within the time required in the protocol, for example over a weekend, or if staff skilled in ECV were not available. Therefore to improve feasibility in the Early ECV 2 Trial, we shall recruit women as early as 33 weeks gestation (see Section 2.12). Additionally, those in the delayed ECV group can have the ECV procedure at any time after 37 weeks (compared to 37-38 weeks in the Pilot). The new scheduling will be more similar to usual clinical practice if early ECV proves to be superior.

- In addition we will encourage centres to establish a systematic, centralised approach to ECV and to implement a policy of routine screening for breech (using Leopold's manoeuvres followed by ultrasound confirmation) at 32-35 weeks gestation to ensure that breech presentations are detected during the gestational age window of eligibility.

\subsection{Timetable for the study}

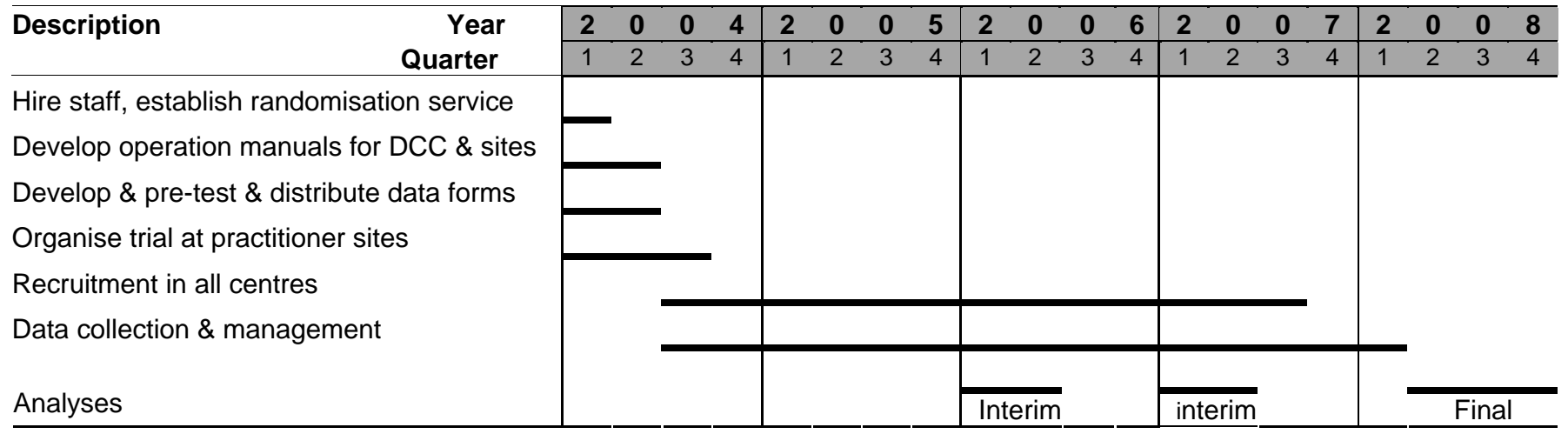

\section{TRIAL MANAGEMENT}

\subsection{Arrangements for day-to-day management of the trial}

The trial will be managed on a day-to-day basis by a small working group which will include Hutton and Study Staff. Data will be managed at the Data Co-ordinating centre at MIRU, where the MIRU director will be responsible for the work. Baseline information will be transmitted to the Coordinating Centre during the randomisation telephone call. Outcome and other data will be collected on NCR paper forms and mailed to the Co-ordinating Centre. Data will be scanned into a TELEform ${ }^{\mathrm{TM}}$ data management system. Logic and range checks will verify the accuracy of the data. Data forms with errors or missing data will be returned to the clinical centres for correction and/or completion. 


\subsection{The role of each applicant}

Hutton is the Principal Investigator and is responsible for the progress and timely completion of the trial. Hutton, Delisle, Carson, Natale, Pollard, Ross and Windrim are responsible for responding to clinical queries, visiting sites, and for encouraging recruitment, protocol compliance and accurate and complete data collection. Hannah is responsible for providing advice based on previous experiences with the co-ordination of multicentre RCTs. Hutton is responsible for the distribution of funds and the administration of the study. Dunn and Ohlsson are responsible for providing advice on neonatal outcomes. Hutton, Hannah, and Willan are responsible for the statistical analyses. Hutton, Hannah, and Gafni are responsible for the economic analyses. All applicants are responsible for assisting with recruitment efforts, including participation in collaborators' meetings, being advocates for the study locally, nationally, and internationally, visiting collaborating centres and attending scientific meetings to present the study results when appropriate.

\subsection{The Steering Committee and the DSMB}

The Steering Committee is responsible for the conduct of the study. The Steering Committee, which includes all applicants, the MIRU Director, the MIRU Research Manager and the Trial Staff, meets every 2-3 months. The DSMB for MIRU trials will be responsible for the review of the interim analyses and for recommending early termination of the study, if necessary, to the Steering Committee. The DSMB currently includes Dr Michael Bracken, Professor, Department of Epidemiology and Public Health, Yale University School of Medicine (chair); Dr Lelia Duley, Resource Centre for Randomised Trials, Nuffield Department of Clinical Medicine, University of Oxford; Prof Allan Donner, Chair, Department of Epidemiology and Biostatistics, The University of Western Ontario; and Dr Patricia Crowley, Coombe Women's Hospital and Trinity College, Dublin. The trial will be conducted according to the Good Clinical Practice guidelines. ${ }^{51}$ 


\section{REFERENCES}

1. Turnbull DA, Wilkinson C, Yaser A, Carty V, Svigos JM, Robinson JS. Women's role and satisfaction in the decision to have a caesarean section. MJA 1999;170:580-3.

2. Geary M, Fanagan M, Boylan P. Maternal satisfaction with management in labour and preference for mode of delivery. J Perinat Med 1997;25(5):433-9.

3. Hildingsson I, Radestad I, Rubertsson C. Waldenstrom U. Few women wish to be delivered by caesarean section. Br J Obstet Gynaecol 2002;109:618-23.

4. Gamble JA, Creedy DK. Women's request for a cesarean section: a critique of the literature. Birth 2000;27(4):256-63.

5. Hannah ME, Hannah WJ, Hewson SA, Hodnett ED, Saigal S, Willan AR for the Term Breech Trial Collaborative Group. Planned caesarean section versus planned vaginal birth for breech presentation at term: a randomised multicentre trial. The Lancet 2000;356:1375-83.

6. Lumley J. Any room left for disagreement about assisting breech births at term? Lancet 2000; 356:1368-9.

7. Shennan A, Bewley S. How to manage term breech deliveries. Br Med J 2001;323:244-5.

8. Hofmeyr GJ, Hannah ME. Planned caesarean section for term breech delivery (Cochrane Review). In: The Cochrane Library, Issue 3, 2003. Oxford: Update Software.

9. Hutton EK, Hannah ME, Barrett J. Use of external cephalic version for breech pregnancy and mode of delivery for breech and twin pregnancy: A survey of Canadian practitioners. J Obstet Gynaecol Canada 2002;24(10):804-10.

10. Minkoff H, Chervenak FA. Elective primary cesarean delivery. N Engl J Med 2003;384(10):946-50.

11. Hall MH, Bewley S. Maternal mortality and mode of delivery. Lancet 1999;354:776.

12. Cooper GM, Lewis G, Neilson J. Editorial: Confidential enquiries into maternal deaths, 1997-1999. Br J Anaesth 2002;89(3):369-72.

13. Waterstone M, Bewley S, Wolfe C. Incidence and predictors of severe obstetric morbidity: case-control study. BMJ 2001;322:1089-94.

14. Health Canada. Canadian Perinatal Surveillance System: Canadian perinatal health report, 2000. Ottawa: Minister of Public Works and Government Services Canada, 2000.

15. Lydon-Rochelle M, Holt VL, Martin DP, Easterling TR. Association between method of delivery and maternal rehospitalization. JAMA 2000;283:2411-6.

16. LaSala AP, Berkeley As. Primary cesarean section and subsequent fertility. Am J Obstet Gynecol 1987;157:379-83.

17. Dashe JS, McIntire DD, Ramus RM, Santos-Ramos R. Twickler DM. Persistence of placenta previa according to gestational age at ultrasound. Obstet Gynecol 2002;99(5 Pt 1):692-7.

18. Lydon-Rochelle M, Holt VL, Easterling TR, Martin DP. First-birth cesarean and placental abruption or previa at second birth. Obstet Gynecol 2001;97(5 Pt 1):765-9.

19. Gilliam M, Rosenberg D, Davis F. The likelihood of placenta previa with greater number of cesarean deliveries and higher parity. Obstet Gynecol 2002;99(6):976-80.

20. Health Canada. Canadian Perinatal Surveillance System: Canadian perinatal health report, 2000. Ottawa: Minister of Public Works and Government Services Canada, 2000.

21. 2000-2001 Canadian institute for Health Information, Reports for Health System Performance, online reports http://secure.cihi.ca/hirpt/jsp/HIDispatcher.jsp

22. Walker R, Turnbull D, Wilkinson C. Strategies to address global cesarean section rates: a review of the evidence. Birth 2002;29(1):28-39.

23. Hofmeyr GJ, Kulier R. External cephalic version for breech presentation at term (Cochrane Review). In: The Cochrane Library, Issue 3, 2003.Oxford: Update Software.

24. Royal College of Obstetricians and Gynaecologists (RCOG) (2001) The management of breech presentation. Green top guidelines no.20, April. London, RCOG. 
25. Hutton EK, Hannah ME, Amankwah K, Kaufman K, Hodnett ED. External cephalic version (ECV) and the Early ECV Trial. J Soc Obstet Gynaecol Can 1999;21(14):1316-26.

26. Hutton EK, Hofmeyr GJ. External cephalic version before term. (Cochrane Review). The Cochrane database Issue 4 2006.

27. Hutton EK, Kaufman K, Hodnett E, Amankwah K, Hewson SA, McKay D, Szalai JP, Hannah ME for the Early External Cephalic Version Trial Group. External cephalic version beginning at 34 weeks gestation versus 37 weeks gestation: a randomized multicenter trial. Am J Obstet Gynecol 2003;189:245-54.

28. Hofmeyr GJ. Effect of external cephalic version in late pregnancy on breech presentation and caesarean section rate: a controlled trial. Br J Obstet Gynaecol 1983;90:392-9.

29. Mahomed K, Seeras R, Coulson R. External cephalic version at term. A randomised controlled trial using tocolysis. Br J Obstet Gynaecol 1991;98:8-13.

30. Hofmeyr GJ, Sadan O, Myer IG, Galal KC, Simko G. External cephalic version and spontaneous version rates: ethnic and other determinants. Br J Obstet Gynaecol 1986;93:13-6.

31. Brocks V, Philipsen T, Secher NJ. A randomized trial of external cephalic version with tocolysis in late pregnancy. Br J Obstet Gynaecol 1984;91:653-6.

32. Van de Pavert R, Gravenhorst JB, Keirse MJNC. Value of external version in breech presentation at term. Ned Tijdschr Geneeskd 1990;134(46):2245-8.

33. Van Dorsten JP, Schifrin BS, Wallace RL. Randomized control trial of external cephalic version with tocolysis in late pregnancy. Am J Obstet Gynecol 1981;141:417-24.

34. Westgren M, Edvall H, Nordstrom L, Svalenius E. Spontaneous cephalic version of breech presentation in the last trimester. Br J Obstet Gynaecol 1985;92:19-22.

35. Healy M, Porter R, Galimberti A. Introducing external cephalic version at 36 weeks or more in a district general hospital: a review and an audit. Br J Obstet Gynaecol 1997;104:1073-9.

36. Myerscough P. Commentary: The practice of external cephalic version. Br J Obstet Gynaecol 1998;105:1043-5.

37. Stedman CM. Discussion: Kornman MT, Kimball KT, Reeves KO. Preterm external cephalic version in an outpatient environment. Am J Obstet Gynecol 1995;172:1738.

38. Hofmeyr GJ. Interventions to help external cephalic version for breech presentation at term (Cochrane Review). In: The Cochrane Library, Issue 3, 2003. Oxford: Update Software.

39. Gyetvai K, Hannah ME, Hodnett ED, Ohlsson A. Tocolytics for preterm labour: a systematic review. Obstet Gynecol 1999;94:869-77.

40. Van Veelen AJ, Van Cappellen AW, Flu PK, Straub MJPF, Wallenburg HCS. Effect of external cephalic version in late pregnancy on presentation at delivery: a randomized controlled trial. British Journal of Obstetrics and Gynaecology 1989;96(8):916-21.

41. Mensink WFA, Huisjes HJ. Is external version useful in breech presentation? Ned T Geneesk 1980; 124(43):1828-31.

42. Hickok DE, Gordon DC, Milberg JA, Williams MA, Daling JR. The frequency of breech presentation by gestational age at birth: a large population-based study. Am J Obstet Gynecol 1992;166: 851-2.

43. Scheer K, Nubar J. Variation of fetal presentation with gestational age. Am J Obstet Gynecol 1976;125(2):269-70.

44. Sorensen T, Hasch E, Lange A. Fetal presentation during pregnancy. The Lancet 1979;2(ii): 477.

45. Hughey MJ. Fetal position during pregnancy. Am J Obstet Gynecol 1985;153:885-6.

46. Lau TK, Stock A, Rogers M. Fetomaternal haemorrhage after external cephalic version at term. Aust NZ J Obstet Gynaecol 1995;35(2):173-4.

47. Cardini F, Weixin H. Moxibustion for correction of breech presentation, a randomised controlled trial. JAMA 1998;280(18):1580-4.

48. Su M, McLeod L, Ross S. Willan A, Hannah WJ, Hutton E, Hewson S, Hannah ME- for the Term Breech Trial Collaborative Group. Factors associated with adverse perinatal outcome in the Term Breech Trial. Am J Obstet Gynecol 2003;189:740-5. 
49. Kramer MS, Demissie K, Yang H, Platt RW, Sauve R, Liston R for the Fetal and Infant Health Study Group of the Canadian Perinatal Surveillance System. The contribution of mild and moderate preterm birth to infant mortality. JAMA2000;284:843-9.

50. Spilker B, Cramer JA. Patient recruitment in clinical trials. Raven Press: New York, 1992.

51. Good Clinical Practice: Consolidated Guideline. Minister of Health, Ottawa, 1997. 